\title{
Histoplasmosis diseminada en paciente inmunocompetentes
}

Daniel Alejandro Valencia Sancho[1], Jacqueline Beatriz Loza Santillan[1], Paulina Elizabeth Santana Vargas[2], Paula Zambrano-Achig[3], Francisco Pérez Tasigchana[3]

1. Médico Internista, Medicina Interna, Hospital de Especialidades Carlos Andrade Marín. Quito. Ecuador.

2. Médico Patólogo, Patología, Hospital de Especialidades Carlos Andrade Marín. Quito. Ecuador.

3. Univesidad UTE, Centro de Investigación en Salud Pública y Epidemiología Clínica (CISPEC). Quito, Ecuador.

Doi: https://doi.org/10.23936/pfr.v5i2.163

PRÁCTICA FAMILIAR RURAL | Vol.5 | No.2 | Julio 2020 | Recibido: 16/04/2020 Aprobado: 15/07/2020

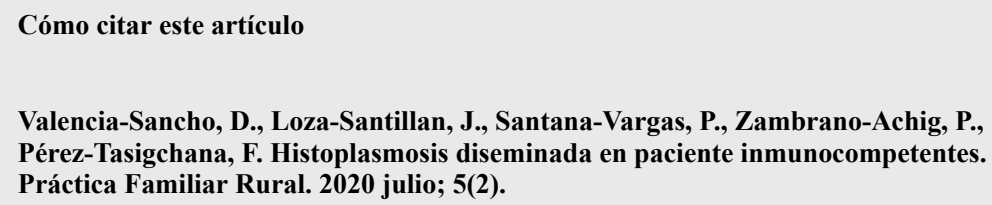

Compartir en:

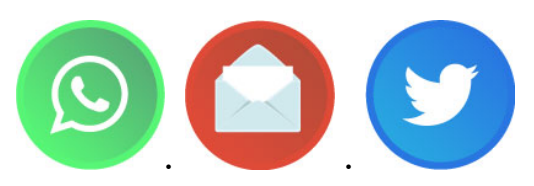

\section{Resumen}

La histoplasmosis es una infección micótica que se adquiere por inhalación causando enfermedad pulmonar en su forma primaria. Puede diseminarse a otros órganos sobre todo en pacientes inmunodeprimidos en quienes alcanza una alta mortalidad. Es endémica en ciertas zonas, en especial las templadas y tropicales. Las manifestaciones clínicas varían según la diseminación y estado inmunológico de la persona. El diagnóstico se lo realiza por la identificación histopatológica, cultivo y serología.

Paciente femenina adulta joven afroamericana sin antecedentes clínicos que presenta cuadro de un año de evolución caracterizado por pérdida involuntaria de peso, alopecia, lesiones dérmicas hiperpigmentadas, diarrea y adenopatías cervicales cuyo estudio histopatológico fue compatible con histoplasmosis. Estudios de extensión sugirieron, pero no confirmaron, una patología autoinmune asociada.

En Ecuador no existe registro de casos de histoplasmosis a pesar de ser considerada una micosis endémica en la zona. El estudio de inmunocompetencia en el caso presente fue sugestivo de un lupus eritematoso sistémico con manifestaciones solapadas. Los pacientes con Lupus 
Eritematoso Sistémico son susceptibles a infecciones oportunistas por hongos dado a defectos inmunológicos intrínsecos con alteración de la inmunidad celular.

La histoplasmosis es una enfermedad endémica en nuestro medio, infradiagnosticada por la diversidad del cuadro clínico y su asociación con otras patologías.

Palabras clave: histoplasmosis, síndrome antifosfolípido, lupus eritematoso sistémico.

\title{
Disseminated histoplasmosis in immunocompetent patients
}

\begin{abstract}
Histoplasmosis is a fungal infection that is acquired by inhalation causing lung disease in its primary form. It can spread to other organs, especially in immunosuppressed patients in whom it reaches high mortality. It is endemic in certain areas, especially temperate and tropical ones. The clinical manifestations vary according to the person's spread and immune status. The diagnosis is made by histopathological identification, culture and serology.

African-American young adult female patient with no clinical history presenting a one-year history characterized by involuntary weight loss, alopecia, hyperpigmented skin lesions, diarrhea and cervical lymphadenopathies whose histopathological study was compatible with histoplasmosis. Extension studies suggested, but did not confirm, an associated autoimmune pathology.

In Ecuador there is no record of cases of histoplasmosis despite being considered an endemic mycosis in the area. The immunocompetence study in the present case was suggestive of systemic lupus erythematosus with overlapping manifestations. Systemic lupus erythematosus patients are susceptible to opportunistic fungal infections due to intrinsic immune defects with impaired cellular immunity.
\end{abstract}

Histoplasmosis is an endemic disease in our environment, underdiagnosed by the diversity of the clinical picture and its association with other pathologies.

Keywords: histoplasmosis, antiphospholipid syndrome, systemic lupus erythematosus

\section{Introducción}

La histoplasmosis clásica es la infección causada por el hongo dimórfico Histoplasma capsulatum var. capsulatum adquirida mediante inhalación de microconidias, que se depositan en los bronquiolos terminales y alvéolos pulmonares, puede presentarse de manera aguda o subaguda causando frecuentemente enfermedad pulmonar pero también puede diseminarse a otros órganos como el bazo, hígado, nódulos linfáticos y médula ósea (1-3).

La infección primaria es adquirida a través de la inhalación provocando respuesta inflamatoria en los pulmones, desde donde se disemina por vía hematológica y usualmente es controlada por la inmunidad mediada por células T1. Puede desarrollarse en los suelos o edificios antiguos enriquecidos con excremento de aves, tras la disrupción humana de los mismos provocando aerosolización de hifas y conidias; o como una saprozoonosis originada por murciélagos (2). Las manifestaciones clínicas dependen tanto del tamaño del inóculo, como del estado inmunológico y las comorbilidades del huésped (3). En caso de enfermedad severa y diseminada la morbilidad es alta (4). La mayoría de pacientes sin inmunodeficiencia son asintomáticos $(5,6)$.

Los casos de histoplasmosis han sido reportados en cada continente excepto en la Antártida. Es de carácter endémico en regiones templadas y tropicales como es Norteamérica se estima que entre el $60 \%$ y el $90 \%$ de 
personas que viven cerca de los valles de los ríos en Ohio y Mississippi han estado expuestos al hongo en algún momento (7), en México en el centro y sur del país hay una prevalencia que va desde el 5\% al 50\% (8). En Panamá también hay un reporte del 50\% de prevalencia (9). En Venezuela va desde el $42.7 \%$ al $85 \%$ $(10,11)$ y en Argentina hay una prevalencia desde el 24.4\% hasta 53.6\% $(12,13)$. En Ecuador no existen datos sobre su prevalencia.

El gold estándar para el diagnóstico es el aislamiento de H. capsulatum en cultivo o la identificación de sus levaduras en histopatológico (14). El cultivo tiene baja sensibilidad y su resultado tarda varias semanas en ser expuesto. La detección de antígenos es sensible, de fácil acceso e interpretación por lo que puede ser un marcador útil para el diagnóstico y seguimiento de respuesta al tratamiento. Los anticuerpos en suero requieren de 4-8 semanas para ser detectados, por lo que son útiles en diagnóstico de formas subagudas y crónicas de histoplasmosis en las que la detección de antígenos es subóptima. Solo en escenarios específicos las pruebas serológicas podrían indicar infección aguda (15). Los métodos moleculares se encuentran en desarrollo, pero hasta el momento su uso clínico no ha sido aprobado por la Agencia de Medicamentos y Alimentos (en sus siglas en ingles FDA). Como en la mayoría de enfermedades infecciosas el diagnóstico óptimo depende de la historia natural de la enfermedad, el sitio donde se desarrolló, la muestra examinada, y el grado de inmunosupresión. El diagnóstico diferencial incluye sarcoidosis, tuberculosis y neoplasias (14).

\section{Caso clínico}

Mujer de 25 años de edad, etnia afroamericana, nacida y residente en Quito, quien presentó desde hace un año compromiso del estado general, decaimiento y pérdida de peso progresiva y significativa de hasta 20 kilos. Ocho meses después, el cuadro se acompañó de diarrea persistente, alopecia, adenopatías cervicales dolorosas la mayor del lado derecho de $6 \mathrm{~cm}$ aproximadamente y lesiones dérmicas hiperpigmentadas maculares diseminadas en tórax, abdomen y espalda. Cuatro meses después manifestó sudoraciones nocturnas y hematemesis con lo cual fue ingresada. Al examen físico se evidenciaron adenopatías cervicales bilaterales móviles y dolorosas a la palpación, una de ellas con salida de exudado blanquecino y de contenido fibrinoide. Auscultación pulmonar y cardíaca normales, abdomen con presencia de hepatomegalia, se constatan lesiones dérmicas descritas. Durante la hospitalización desarrolla lesiones ulcerativas en paladar blando y carrillos dentarios (Figura 1). Los estudios de laboratorio mostraron una anemia microcítica hipocrómica, proteínas totales y albúmina disminuidas, las pruebas de función renal, enzimas hepáticas, glucosa en ayunas y HbA1c fueron normales. El IgM para citomegalovirus fue negativo, Virus de Inmunodeficiencia Humana (VIH) cuarta generación negativo por dos ocasiones aisladas. La ecografía abdominal mostró una hepatomegalia de $16.8 \mathrm{~cm}$. El perfil inmunológico y anticuerpos antifosfolípidos fueron negativos con excepción del Coombs directo, ANA con reactividad 1/80 y el anticoagulante lúpico que fue débilmente positivo. La proteinuria en orina de 24 horas fue de $326 \mathrm{mg} / \mathrm{dl}$ y la electroforesis de proteínas mostró policlonalidad. El estudio de inmunoglobulinas séricas estuvo dentro de normalidad. Se realizó biopsia abierta de adenopatía cervical evidenciándose células en cuyo citoplasma se observan microorganismos HE, PAS y GROCOTT positivos tipo levadura compatibles con histoplasma. (Figura 2). Se realizaron 2 serologías VIH de cuarta generación que fueron negativas. La fibrobroncoscopia no aisló gérmenes, el GeneXpert tuberculosis multidrogo resistencia fue negativo. Fue ingresada para recibir Anfotericina B, y a las 48 horas de hospitalización presentó clínica de una tromboembolia pulmonar aguda misma que fue confirmada mediante angiotomografía pulmonar, por lo cual fue anticoagulada. Las tomografías corporales fueron negativas para lesiones sugestivas de tumoraciones. El mielocultivo fue negativo y no reportó infiltrados neoplásicos. En el estudio endoscópico se evidenció pólipos duodenales, no sugestivos de malignidad. En el ecocardiograma transesofagico se observó una masa móvil con centro hipodenso de bordes regulares de $28 \times 13 \mathrm{~mm}$, sugestivo de trombo vs masa extracardiaca.

Como dato pertinente respecto al posible lugar de contagio, desde hace 2 años la paciente ha viajado de manera intermitente a una zona tropical rural-agropecuaria en Quevedo - Los Ríos en donde se encontraba en contacto con animales de granja incluyendo varias especies avícolas.

Figura 1. Úlceras bucales localizadas en paladar superior. 

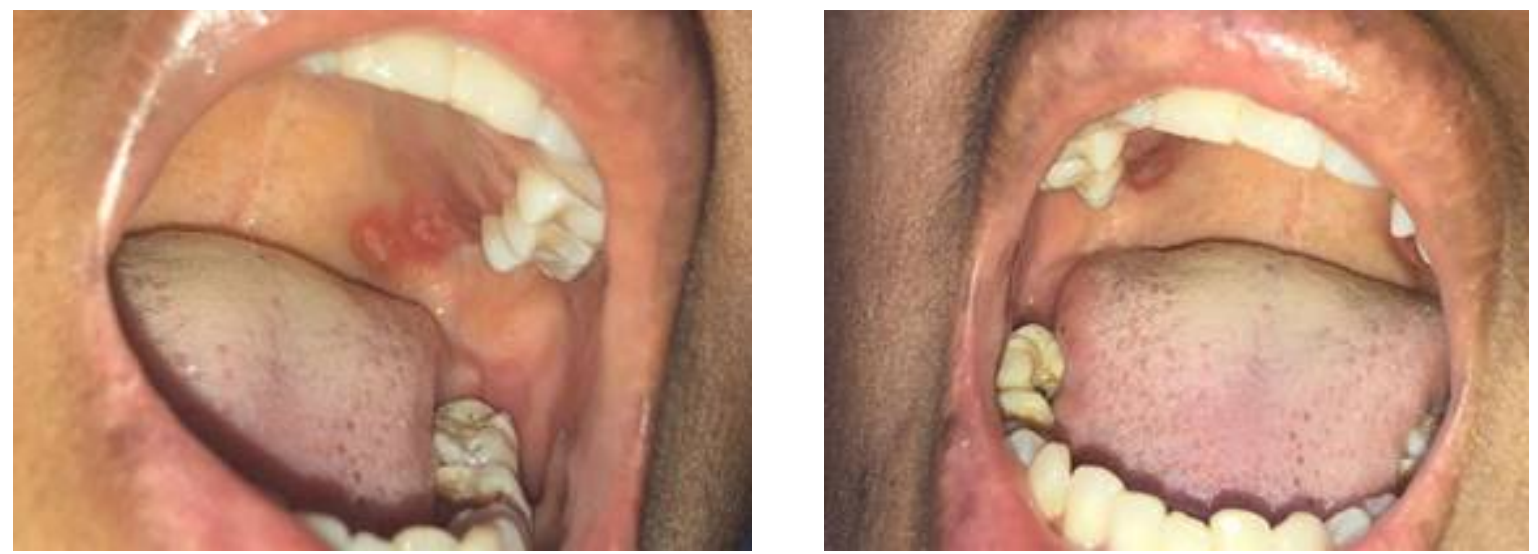

Figura 2. 1A. 1B. 1C. 1D.

$1 \mathrm{~A}$ $1 \mathrm{~B}$
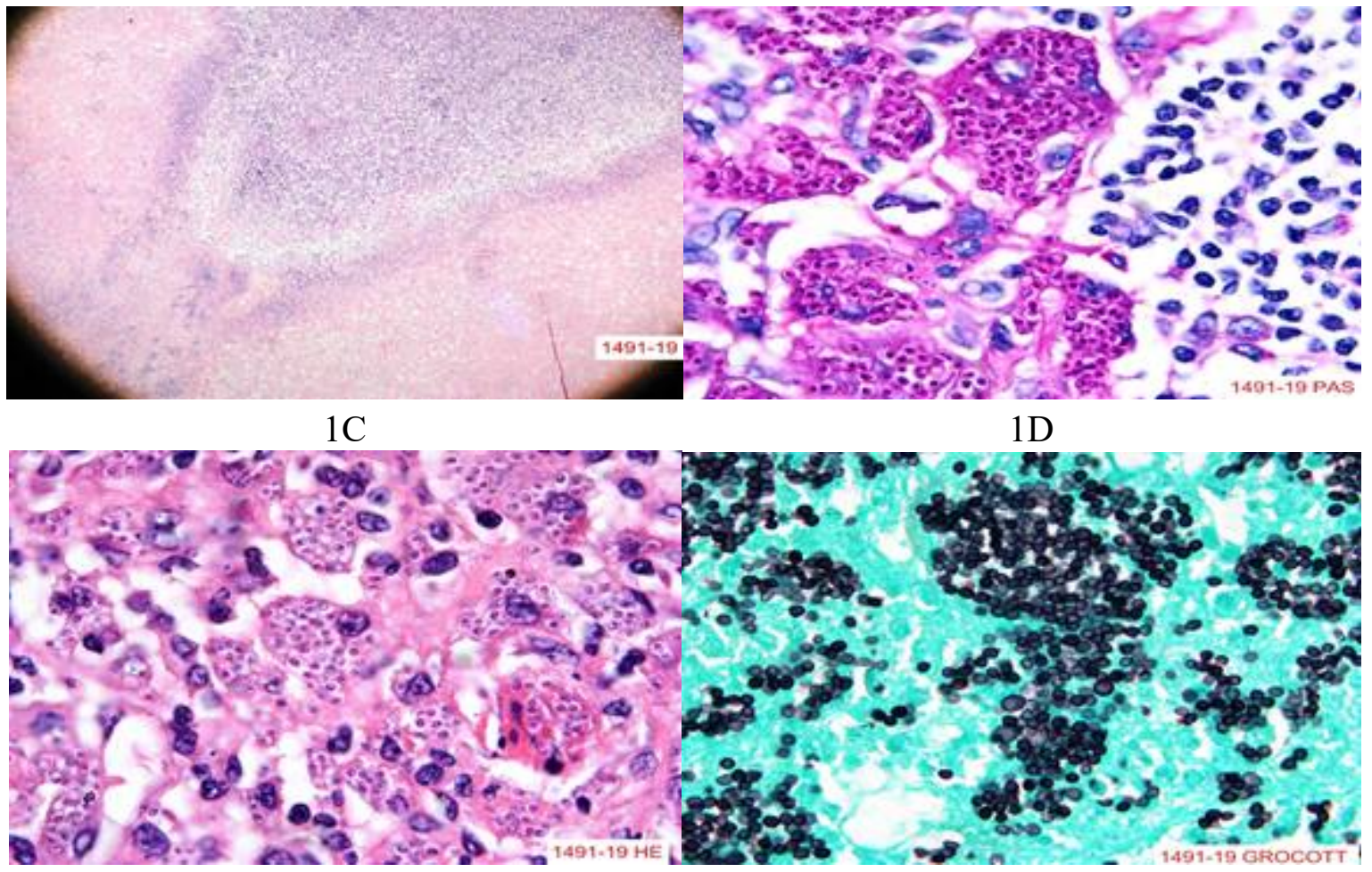

Los cortes muestran ganglios linfáticos con distorsión de arquitectura con extensas áreas de necrosis y marcado infiltrado de histiocitos con núcleos sin atipia cromatina fina con abundante citoplasma en el cual se aprecia gran cantidad de microorganismos ovalados tipo levaduras compatible con histoplasma, además se aprecian células gigantes multinucleadas

Histoquímica: PAS, GROCOTT: Positivos en microorganismos.

\section{Discusión}

La historia de esta micosis comienza en 1906 con los importantes hallazgos realizados por Darling a partir de la necropsia de un hombre de 27 años, que trabajaba en las obras de construcción del canal de Panamá; describió al microorganismo observado como "un parásito de forma ovoide a redonda, rodeado de un halo que no se colorea, con un grosor de aproximadamente 1/6 del diámetro del parásito" (15). La mayor epidemia conocida de histoplasmosis tuvo lugar en Indianápolis, Estados Unidos (16). En 1998 se publicaron 3 casos importados: 2 pacientes de Guinea ecuatorial, Ecuador y un tercero que había viajado por la selva de Perú (17). Aunque según los datos del Instituto Nacional Ecuatoriano de Censo, la cantidad exacta de casos de histoplasmosis en Ecuador no está establecida, es bien conocido que vivimos en un área 
endémica para este tipo de micosis tanto en la Costa como en la Amazonia por las condiciones demográficas y la fauna existente. En el caso de nuestra paciente, la presunta fuente de infección es en Quevedo en una zona rural. La presencia de aves y murciélagos en nuestra región costera, el clima templado cálido con alta humedad por la presencia del mar y neblinas frecuentes, además de los suelos ácidos por las plantaciones forestales de Pinus y Eucalyptus que rodean el litoral ecuatoriano, aportan las condiciones favorables para la adaptación de este hongo, haciéndolo ubicuo y muy frecuente (18).

Para Staffolani y cols, los casos de histoplasmosis en inmunocompetentes son infra diagnosticados e infra reportados, ya que por lo general se presentan de manera asintomática o con síntomas leves y auto limitados ( $95 \%$ de los casos). La infección puede producirse tanto de novó o por reactivación incluso años después del contagio. La diseminación se desarrolla por lo general en casos de inmunosupresión manifiesta, sin embargo, su presencia en pacientes sin compromiso inmune se debe mantener abierta (19). En el presente caso descrito se investigaron todos los posibles factores de riesgo relacionados con la diseminación de la infección: neoplasias sobre todo oncohematológicas, diabetes, neumopatía crónica, infección por Virus de Inmunodeficiencia Humana, infección activa por citomegalovirus, uso crónico de esteroides, enfermedades inmunológicas, trasplante de órganos (20). Todas estas patologías fueron descartadas, excepto las autoinmunes que, aunque aún no hay confirmación, existe una posibilidad abierta de la presencia de Lupus Eritematoso Sistémico. Al no encontrar ninguna enfermedad subyacente que cause una disfunción de la inmunidad mediada por células $\mathrm{T}$, se trata de un caso poco común, ya que por fisiopatología la diseminación de la enfermedad depende de este hecho. La posibilidad de una inmunodeficiencia innata, que también podría ser una causa de diseminación de la infección, no fue considerada en el caso ya que dentro de su historia personal se trata de una paciente en cuya niñez y adolescencia no se reportan infecciones a repetición o de localización profunda, abscesos cutáneos recurrentes, uso prolongado de antibioticoterapia o necesidad de los mismos por vía intravenosa o aftas persistentes (21). Igualmente, su historia familiar no mostró datos relevantes.

La distribución epidemiológica habitual de la histoplasmosis también contrasta con el caso en estudio, ya que se conoce que compromete más a hombres que a mujeres con una relación $4: 1$, puede presentarse a cualquier edad, aunque con más frecuencia en niños y mayores de 50 años (22).

El desarrollo de una hemoptisis por tromboembolia pulmonar a las 48 horas de hospitalización, además de la presencia de lesiones sugestivas de un trombo que nace de vena cava a nivel cardíaco nos incita a considerar la posibilidad de un trastorno de hipercoagulabilidad de base que en este caso encajaría con un Síndrome Antifosfolipídico. El perfil laboratorial del mismo fue negativo, y no se encontró ningún tipo de neoplasia o hemopatía maligna. Igualmente se sospechó una colagenopatía, como criterios clínicos y laboratoriales presentó alopecia, úlceras bucales no sugestivas de otras patologías, un Coombs directo positivo, anticuerpos antinucleares con reactividad ligera, anticoagulante lúpico débil positivo. Se observó además la presencia de una linfopenia transitoria y un derrame pleural bilateral con remisión espontánea y casi inmediata por lo que no fueron considerados como criterios. El hecho de que el anticoagulante lúpico fue positivo abre la posibilidad de un síndrome antifosfolipídico cuyo diagnóstico deberá establecerse con una nueva positivización de uno de los anticuerpos antifosfolipídicos 12 semanas después tal como lo indican los criterios diagnósticos de Sapporo (23). Aunque la combinación de síntomas, signos y alteraciones inmunológicas no reunieron criterios diagnósticos suficientes como para una diagnosticar una colagenopatía tipo Lupus Eritematoso Sistémico (LES), no se puede descartar de manera definitiva esta patología. Es pertinente considerar que el que Síndrome Antifosfolípido (SAF) puede ser secundario a LES en un 30-40\% de los casos (18). En una serie prospectiva de 1000 pacientes con SAF descrita por el Euro-Phospholipid Projet Group se observaron 53,1 \% de SAF primarios, un 36,2 \% asociados a LES, un 5\% asociados a síndrome lupuslike (23). El estudio de Freire PV y cols. analizó de manera retrospectiva una cohorte de 80 pacientes con síndrome antifosfolipídico en este caso primario de las cuales el $17.5 \%$ progresaron en un periodo de 5 +/- 2 años a síndrome antifosfolipídico asociado a LES (18).

Datos recientes han sugerido que una variante específica de genes que codifican una proteína llamada lectina que une manosa (MBL del inglés mannose-binding lectin) y receptores Fcg podrían aumentar el riesgo de infecciones oportunistas en pacientes con LES8,9. MBL es una proteína sérica que juega un rol importante en la defensa inmunológica, particularmente durante la fase de contacto primaria entre el patógeno y el organismo. Ciertos polimorfismos genéticos que producen niveles bajos o deficiencias de MBL están 
asociados con un incremento en la susceptibilidad para infecciones y para el riesgo de desarrollar LES10,11. Sin embargo, niveles bajos de MBL pueden ser encontrados en 4 a 10\% de la población adulta sana. Garred y cols, reportaron una incidencia de infecciones cuatro veces mayor en pacientes con LES que fueron homocigóticos para la variante alélica del MBL.

En la historia natural del LES existe un periodo subclínico, seguido de una fase clínica con el debut de los síntomas y signos. La fase entre el debut clínico y el diagnóstico puede encajar con una enfermedad indiferenciada del tejido conectivo (24). Los estudios de cohortes muestran que en la historia natural del LES, la demora entre el inicio clínico de la enfermedad y su diagnóstico se ha ido reduciendo con el pasar de los años y la mejora de pruebas de laboratorio, pasando de hasta 26 meses en los pacientes diagnosticados en la década de los 80 a 15 meses en la década de los 90 y a nueve meses a partir del año 2000 (25). Con esto, no es improbable que exista un solapamiento de una enfermedad autoinmune ya presenté Adicionalmente desde el punto de vista epidemiológico, habría que considerar la relación 10:1 mujer-hombre y que en pacientes afroamericanos (como la del caso en mención) la prevalencia es de 200 casos por 100.000 habitantes en comparación con otras etnias (sobretodo blanco caucásica) que es de 40 casos por 100.000 habitantes (26).

\section{Conclusiones}

La histoplasmosis diseminada es una enfermedad no reportada en nuestro país y por tal motivo probablemente infradiagnosticada. Ante la presencia de la misma es obligatorio el estudio de inmunocompromiso de base, que justifique la extensión de esta micosis como en el presente caso.

\section{Conflictos de interes}

Los autores declaran no presentar ningún conflicto de interés respecto a la presentación de este caso clínico.

\section{Agradecimientos}

Al personal de salud del servicio de Medicina Interna e Infectología del HECAM.

\section{Referencias bibliográficas:}

1. Bahr NC, Antinori S, Wheat LJ, Sarosi GA. Histoplasmosis infections worldwide: thinking outside of the Ohio River valley. Curr Trop Med reports. 2015;2(2):70-80.

2. Seyedmousavi S, Guillot J, Tolooe A, Verweij PE, de Hoog GS. Neglected fungal zoonoses: hidden threats to man and animals. Clin Microbiol Infect. 2015;21(5):416-25.

3. Staffolani S, Buonfrate D, Angheben A, Gobbi F, Giorli G, Guerriero M, et al. Acute histoplasmosis in immunocompetent travelers: a systematic review of literature. BMC Infect Dis. 2018;18:673.

4. Wheat L, Azar M, Bahr N, Spec A, Relich R, Hage C. Histoplasmosis. Infect Dis Clin N Am. 2016;30(1):207-27.

5. Álvarez E, Amaro J, Villavicencio L. Histoplasma capsulatum:¿ un agente emergente para Chile?. Rev Chil infectología. 2018;35(3):309-11.

6. Scully MC, Baddley JW. Epidemiology of histoplasmosis. Curr Fungal Infect Rep. 2018;12(1):51-8.

7. Manos N, Ferebee S, Kerschbaum W. Geographic variation in the prevalence of histoplasmin sensitivity. Dis chest. 1956;29(6):649-68.

8. Laniado-Laborin R. Coccidioidomycosis and other endemic mycoses in Mexico. Rev Iberoam Micol. 2007;24:249-58.

9. Gutierrez M, Canton A, Sosa N, Puga E, Talavera L. Disseminated histoplasmosis in patients with AIDS in Panama: a review of 104 cases. Clin Infect Dis. 2005;40:1199-202.

10. Mata-Essayag S, Colella M, Rosello A. Histoplasmosis: a study of 158 cases in Venezuela, 2000 - 2005. Medicine (Baltimore). 2008;87:193-202.

11. Cermeno J, Hernandez I, Cermeno J. Epidemiological survey of histoplasmine and paracoccidioidine skin reactivity in an agricultural area in Bolivar state, Venezuela. Eur J Epidemiol. 2004;19:189-93.

12. Van Gelderen de Komaid A, Duran E. Histoplasmosis in northwestern Argentina. II: Prevalence of Histoplasmosis capsulati and paracoccidioidomycosis in the population south of Chuscha, Gonzalo and 
Potrero in the province of Tucuman. Mycopathologia 1995; 129 : 17 - 23. Mycopathologia. 1995;129:1723.

13. Van Gelderen de Komaid, A Duran E, Borges de Kestelman I. Histoplasmosis and paracoccidioidomycosis in northwestern Argentina III. Epidemiological survey in Vipos, La Toma, and Choromoro - Trancas, Tucuman, Argentina. Eur J Epidemiol. 1999;15:383-8.

14. Azar M, Hage C. Laboratory diagnostics for histoplasmosis. J Clin Microbiol. 2017;55:1612-20.

15. Dufresne SF, Marr KA, Shoham S. Diagnosis of Systemic Fungal Diseases. In Principles and Practice of Transplant Infectious. Diseases. 2019;819-40.

16. Morán Ayala MP. Determinación de histoplasmosis en aspirado de médula ósea en pacientes con VIH en el Hospital de Infectología". Universidad de Guayaquil; 2015.

17. Molina L, Garau M, García J, Odriozola M, Del Palacio A. A propósito de tres casos de histoplasmosis importada en nuestro hospital. Rev Iberoam Micol. 2002;19:204-7.

18. Bueter C, Deepe GS, Rappleye CA. Histoplasma capsulatum and Histoplasmosis. Curr Prog Med Mycol. 2017;139-67.

19. Gajurel K, Dhakal R, Deresinski S. Histoplasmosis in transplant recipients. Clin Transpl. 2017;31(10):13087.

20. Cáceres DH, Gómez BL, Restrepo Á, Tobón ÁM. Histoplasmosis y sida: factores de riesgo clínicos y de laboratorio asociados al pronóstico de la enfermedad. Infection. 2012;16:44-50.

21. Pedraza Á, Vargas-Rumilla, M. I Ramírez-Roa JL. Registry of primary immunodeficiencies in children at a fourth level hospital. Bogota, 2010-2016. Rev Alerg Mex. 2018;65(4):341-8.

22. Almeida MA, Almeida-Silva F, Guimarães AJ, Almeida-Paes R, Zancopé-Oliveira RM. The occurrence of histoplasmosis in Brazil: A systematic review. Int J Infect Dis. 2019;86:147-56.

23. Cervera R. Antiphospholipid syndrome. Thrombosis research, 151, S43-S47. Thromb Res. 2017;151:s43-7.

24. Hedrich CM, Smith EM, Beresford MW. Juvenile-onset systemic lupus erythematosus (jSLE)Pathophysiological concepts and treatment options. Best Pract Res Clin Rheumatol. 2017;31(4):488-504.

25. Cervera R, Serrano R, Pons-Estel GJ, Ceberio-Hualde, L Shoenfeld Y, De Ramón E, Tincani A. Morbidity and mortality in the antiphospholipid syndrome during a 10-year period: a multicentre prospective study of 1000 patients. Ann Rheum Dis. 2015;74(6):1011-8.

26. Ministerio de Salud Pública del Ecuador Lupus Eritematoso Sistémico (LES). Guía de Práctica Clínica (Adopción GPC LES chilena) Quito: Ministerio de Salud Pública, Dirección Nacional de NormatizaciónMSP. 2016. 